\title{
Inteligencia emocional y salud en el envejecimiento: beneficios del programa PECI-PM ${ }^{*}$
}

\author{
Emotional Intelligence and Health in Aging: PECI-PM \\ Program Benefits
}

\author{
María del Carmen Pérez-Fuentes ${ }^{1}$ \\ José J. Gázquez Linares ${ }^{2}$ \\ María del Mar Molero Jurado ${ }^{3}$ \\ África Martínez ${ }^{4}$ \\ Ana Belén Barragán Martín ${ }^{5}$ \\ María del Mar Simón Márquez ${ }^{6}$ \\ Universidad de Almería, España
}

\begin{abstract}
Resumen. El entrenamiento de la inteligencia emocional en personas mayores, ha demostrado su efectividad en la mejora de la calidad de vida. El objetivo es analizar los beneficios en salud, de un programa de estimulación cognitiva e inteligencia emocional para personas mayores (PECI-PM). La muestra estuvo formada por 28 alumnos de la Universidad de Mayores de Almería, que completaron la primera fase del PECI-PM. Para la evaluación (pre-post) de la salud, se aplicó la versión española del SF-36. Los resultados mostraron puntuaciones significativamente más elevadas, tras la intervención, en las dimensiones de salud: Función Social, Dolor Corporal, Vitalidad y Función Social. La primera implementación del PECIPM, revela efectos positivos del entrenamiento emocional sobre la percepción de la salud, en personas mayores.
\end{abstract}

Palabras clave. Inteligencia emocional, cognición, salud, mayores, PECI-PM.

Abstract. Training emotional intelligence in the elderly, it has demonstrated its effectiveness in improving the quality of life. The aim is to analyze the health benefits of a program of cognitive stimulation and emotional intelligence for older people (PECI-PM). The sample consisted of 28 students of the University Senior Almeria, who completed the first phase of PECI-PM. For the (pre-post) assessment of health, the Spanish version of the SF-36 was applied. The results showed significantly higher scores after the intervention, the dimensions of health: social function, bodily pain, vitality and social function. The first implementation of the PECI-PM reveals positive effects of emotional training on perceived health in the elderly.

Keywords. Emotional intelligence, cognition, health, elderly, PECI-PM.

\footnotetext{
${ }^{1}$ María del Carmen Pérez-Fuentes. Universidad de Almería. España. Dirección postal: Ctra. de Sacramento, s/n (04120) Almería - España E-mail: mpf421@ual.es

2José J. Gázquez Linares. Universidad de Almería. España. E-mail: jlinares@ual.es

${ }^{3}$ María del Mar Molero Jurado. Universidad de Almería. España. E-mail: mmj130@ual.es

${ }^{4}$ África Martínez. Universidad de Almería. España. E-mail: amm521@ual.es

${ }^{5}$ Ana Belén Barragán Martín. Universidad de Almería. España. E-mail: abm410@ual.es

${ }^{6}$ María del Mar Simón Márquez. Universidad de Almería. España. E-mail: abm410@ual.es
}

*Este trabajo cuenta con la colaboración del Proyecto Almería Urban, cofinanciado por fondos FEDER y el Ayuntamiento de Almería.

\section{(@) $\Theta \Theta \Theta$}

Esta obra está bajo una licencia de Creative Commons Reconocimiento-NoComercial-SinObraDerivada 4.0 Internacional. 


\section{Introducción}

En la actualidad, los cambios sociodemográficos, y la evolución en las necesidades, actitudes e intereses de la población que envejece (Gázquez, PérezFuentes, Mercader, \& Molero, 2011), requieren de una revisión del modelo de salud y los factores asociados (Organización Mundial de la Salud, 2015). Hasta hace tan sólo unos años, el interés sobre la salud de la población mayor se centraba fundamentalmente en los procesos de enfermedad y en los factores de riesgo (Gonzalo \& Pasarín, 2004). No obstante, desde el paradigma del Envejecimiento Activo, como marco de referencia (Lopesino, López-Ceron, Muñoz, y Echanagorría, 2011), la atención exclusiva hacia los factores de riesgo asociados a la enfermedad resulta insuficiente. Por tanto, se plantea la necesidad de reorientar el interés de la investigación hacia los factores que influyen positivamente, no solo en la prevención de la enfermedad, sino en la promoción de la salud (Ramírez, Ortega, \& Martos, 2015).

Entre las diferentes líneas de actuación en envejecimiento activo, las Universidades de Mayores se presentan como una de las iniciativas con mayor auge en España. La participación en este tipo de actividades, además de restar valor a los estereotipos sobre la vejez (Gázquez et al., 2009), tiene una repercusión positiva en la integración social, en la salud física y mental de los alumnos (Gázquez, Pérez-Fuentes, \& Carrión, 2010), así como en el mantenimiento de las funciones cognitivas (Plaza, Requena, Rosario, \& López, 2015). De los beneficios derivados de las diferentes iniciativas para la mejora de la calidad de vida en personas mayores, se extrae la necesidad de identificar nuevos elementos para el diseño y la promoción de intervenciones eficaces (Díaz-Prieto \& García-Sánchez, 2015).

Por otro lado, es importante señalar el valor que las personas mayores otorgan a la salud, como una de las dimensiones más relevantes de su calidad de vida (Fernández-Ballesteros et al., 2010), además del peso determinante que tiene sobre la percepción del propio proceso de envejecimiento (Pérez-Fuentes et al., 2015). En los últimos años, se ha prestado especial interés al bienestar emocional, en el ajuste psicosocial durante todas las etapas de la vida (Salguero, FernándezBerrocal, Ruiz-Aranda, Castillo, \& Palomera, 2011), y a la forma en que éste puede contribuir a un envejecimiento óptimo (Arias \& Iglesias-Parro, 2015).

El concepto de inteligencia emocional, fue utilizado como tal por Salovey y Mayer (1990) para hacer referencia a las habilidades y capacidades para percibir y producir emociones que posibiliten el pensamiento, para entender esas emociones y regularlas, con una clara función adaptativa del individuo.

En 1991, Lazarus hace referencia a la valoración de un estímulo externo o de una situación, como uno de los factores cognitivos más influyentes. Según este autor, tendrían lugar dos procesos de valoración: uno que ocurre de forma automática, y otro que se produce conscientemente. Así, cada emoción se evoca por un patrón distinto de valoración, por tanto, los estados emocionales se pueden diferenciar según los componentes de evaluación que estén implicados, en cada caso (Smith \& Lazarus, 1993).

Goleman (1995), pone de relieve la importancia de estudiar tanto la inteligencia como las emociones de forma conjunta, reforzando el significado de "ser inteligente" y otorgando mayor peso a las emociones en el desarrollo vital del ser humano. Posteriormente, en 1998, Goleman expone que las personas con mayor nivel de inteligencia emocional serán también más sanas, felices y productivas en su vida diaria. En esta línea, también cobran sentido otras propuestas como la de LeDoux (1996) que, desde el contexto de las teorías multinivel, reconoce la complejidad del sistema cognitivo, donde las reacciones emocionales pueden ser desencadenadas por procesos preatencionales o automáticos.

Por su parte, Bar-On (1997) utiliza la expresión "inteligencia emocional y social" para hacer referencia a las habilidades y capacidades (personales, emocionales y sociales) que facilitan la adaptación e interacción con el medio. Desde el modelo de Bar-On, este tipo de inteligencia será la que facilite el éxito social y bienestar psicológico, a lo largo del ciclo vital, con efectos significativos sobre la salud física y mental (Bar- 
On, 2006). Precisamente, será una adaptación para personas mayores (Pérez-Fuentes, Gázquez, Mercader, \& Molero, 2014) del instrumento desarrollado originalmente por el autor (Emotional Quotient Inventory EQ-i; Bar-On, 1997), la que fue considerada durante la elaboración del PECI-PM y utilizada en la valoración de los cambios en las dimensiones de Inteligencia Emocional, tras la intervención (Pérez-Fuentes, Molero, Gázquez, \& Soler, 2014).

Hoy día, se sabe que la capacidad para regular las emociones se mantiene estable, o incluso puede mejorar con la edad, y que habilidades relacionadas con el uso del humor pueden resultar una estrategia eficaz (Harm, Vieillard, \& Didierjean, 2014). En otros casos, se ha incluido la función cognitiva en el análisis de las habilidades emocionales (Broche, Rodríguez, \& Martínez, 2014). Suzuki y Akiyama (2013), examinaron la influencia del envejecimiento cognitivo sobre el reconocimiento de expresiones faciales en las emociones básicas. Encontraron, que el deterioro cognitivo relacionado con la edad, afectaba negativamente al reconocimiento de emociones, como la alegría, la sorpresa, el miedo, la ira, el asco y la tristeza. Una prueba más de que los procesos cognitivos, no funcionan de manera aislada (Clemente, García-Sevilla, \& Méndez, 2015; Sanz de Acedo y Sanz de Acedo, 2013).

A pesar delaemoción y la cognición, tradicionalmente, han sido considerados como aspectos independientes, la evolución teórica de la inteligencia emocional, y a pesar de tratarse de un concepto relativamente joven, apoya la existencia de una interrelación de ambos procesos. Aunque la inteligencia emocional ha demostrado estar relacionada positivamente con el bienestar, la salud mental y física, todavía sigue abierto el debate sobre los procesos cognitivos subyacentes (Gutiérrez-Cobo, Cabello, \& Fernández-Berrocal, 2016). Precisamente, de estas cuestiones sin resolver se derivan algunas de las dificultades identificadas en la medida de las dimensiones de inteligencia emocional. Muestra de ello es, por ejemplo, el efecto que tiene el formato de respuesta (verbal/ imagen) en la medición de la comprensión emocional (Delgado, 2016).

\section{Inteligencia emocional y funcionamiento social}

La emoción no puede ser concebida únicamente como un fenómeno intrapersonal (Rimé, Mesquita, Boca, \& Philippot, 1991). El intercambio social de experiencias es un componente fundamental en la configuración de los procesos emocionales del individuo (Azpiazu, Esnaola, \& Sarasa, 2015; Del Barrio et al., 2011). Así, trabajos como el de Krawczyk, Lelek, Moroz, Kamenczak y Maj (2009), ponen de manifiesto que la mejora en aquellas capacidades que permitan el uso adecuado de la inteligencia emocional en la resolución de problemas y el funcionamiento social, serán un factor clave de programas terapéuticos eficaces. Este es el caso de trastornos como la Fobia Social Generalizada, caracterizada por un temor excesivo a las situaciones sociales.

Las personas que padecen este trastorno presentan un sesgo en la interpretación social, por tanto, dificultad para percibir, comprender y manejar las emociones. Al respecto, Jacobs et al. (2008), encontraron una correlación negativa entre el nivel de ansiedad social y la inteligencia emocional, en pacientes con Fobia Social Generalizada.

Por su parte, Lakey y Orehek (2011) afirman que el apoyo percibido es un factor asociado a un buen estado de salud mental, cumpliendo una función amortiguadora del estrés. En estudios previos (Schmidt \& Andrykowski, 2004), ya se planteaba la posibilidad de que un alto nivel de inteligencia emocional, podría reducir el impacto negativo de un ambiente social dañino. De manera que, un adecuado procesamiento cognitivo y emocional, facilita un ajuste positivo del individuo ante experiencias traumáticas relacionadas con la enfermedad.

La inteligencia emocional implica la interacción entre el individuo y su entorno social más amplio. Así, hacer frente a las situaciones adversas, requiere del desarrollo de la capacidad de recuperación emocional (Schneider, Lyons, \& Khazon, 2013), pudiendo desarrollarse ésta con el apoyo social necesario (Armstrong, Galligan, \& Critchley, 2011). En la actualidad, avalando el papel de lo social en la salud de las personas de edad 
avanzada, continúa abierto el debate donde se discute la evidencia empírica que avala los efectos beneficiosos de las relaciones sociales para la salud (Iglesias-Parro \& Arias, 2015).

\section{La inteligencia emocional en los procesos de salud-enfermedad $y$ percepción del dolor}

La regulación de las emociones tiene consecuencias importantes para la salud. En esta línea, existen intervenciones eficaces que entrenan habilidades de regulación emocional para la mejora de la salud, tanto en poblaciones de riesgo como en individuos que ya han sido diagnosticados (Jazaieri, Morrison, Goldin, y Gross, 2015; Quoidbach, Mikolajczak, \& Gross, 2015; Zeidner, Matthews, \& Roberts, 2012).

La inhibición de la expresión emocional ha sido asociada con la incidencia y progresión de enfermedades crónicas, como el trastorno cardiovascular (Montero, Rueda, \& Bermúdez, 2014). Las emociones negativas se han asociado a un aumento de las tasas de muerte cardiovascular y a incidentes cardíacos recurrentes (Smith \& Blumenthal, 2011). Por los efectos que tiene en la calidad de vida de pacientes y familiares, también en las enfermedades neurodegenerativas, se ha examinado el papel de la emoción y su relación con el estado de las funciones cognitivas. Bucks y Radford (2004), observaron que, a pesar del deterioro en la capacidad cognitiva general, el reconocimiento de señales emocionales en la expresión facial en pacientes con Alzheimer se encontraba relativamente conservado. Más recientemente, Alonso-Recio, Serrano-Rodríguez, Carvajal-Molina, Loeches-Alonso, y Martín-Plasencia (2012), llevan a cabo una revisión sobre el reconocimiento de la expresión facial de emociones en la enfermedad de Parkinson. Aunque, la mayoría de los estudios sugieren la existencia de deficiencias en el reconocimiento de emociones, es posible que dichas alteraciones estén relacionadas con el deterioro cognitivo propio de la enfermedad.

Pero, el papel de las emociones no solo se limita al curso y/o sintomatología de una enfermedad, sino que también ha sido estudiado en relación a la práctica de determinados hábitos relacionados con la salud
(Saklofske, Austin, Galloway, \& Davidson, 2007). La falta de sueño puede afectar a una gran variedad de funciones cognitivas, pero ¿qué efectos tiene sobre la inteligencia emocional percibida? Respecto a esta cuestión, Killgore et al. (2008), encontraron que la privación de sueño se asoció con puntuaciones más bajas en inteligencia emocional, provocando además cambios temporales en el metabolismo cerebral, la cognición y el comportamiento. En otros casos (McKenna, 2007), se ha hecho referencia a la actitud hacia conductas saludables como la práctica de ejercicio físico, donde la inteligencia emocional ha sido relacionada positivamente con este hábito.

En cuanto al efecto de las emociones en la percepción/regulación del dolor, existen trabajos recientes que apuestan por el papel modulador de los factores emocionales en enfermedades crónicas (Bushnell, Ceko, \& Low, 2013). Según Rhudy y Meagher (2001), las emociones positivas atenúan la sensación de dolor, mientras que la intensidad de dolor percibido puede llegar a intensificarse con las emociones negativas. En otros casos, se han identificado estructuras cerebrales implicadas, relacionadas a su vez con el aprendizaje emocional y la memoria (Apkarian, 2008; Villemure y Bushnell, 2009). De hecho, en relación a ésta última, recientemente se argumenta sobre el efecto del envejecimiento sobre la habilidad para reconocer información previamente procesada (Ruiz, Suengas, Simón, \& Pastor, 2015).

\section{Inteligencia emocional y salud mental}

El déficit en la percepción y regulación emocional se ha estudiado como uno de los objetivos de tratamientos en diferentes trastornos mentales (Garrido-Vásquez, Jessen, \& Kotz, 2011). Para Berking y Wupperman (2012), la dificultad para hacer frente a las emociones es una característica común en los pacientes con sintomatología depresiva, en el trastorno límite de la personalidad, en los problemas con el consumo de sustancias y en trastornos somatomorfos, entre otros. Por otro lado, los resultados obtenidos por diversos autores (Hughes \& Gullone, 2011; Markey \& Vander Wal, 2007; Watson, Steele, Bergin, Fursland, \& Wade, 2011), ponen de manifiesto el papel de la emoción en el 
desarrollo y mantenimiento de trastornos relacionados con la alimentación, y en particular, sobre la dificultad de estos pacientes para regular las emociones.

Por otro lado, los trastornos del estado de ánimo han sido también objeto de estudio en población mayor (Cardila et al., 2015; Gázquez, Pérez-Fuentes, Lucas, \& Yuste, 2008), atendiendo al papel de la inteligencia emocional en el desarrollo y/o mantenimiento de la sintomatología (Lombas, Martín-Albo, ValdiviaSalas, \& Jiménez, 2014). Hansenne y Bianchi (2009), revelan niveles más bajos de inteligencia emocional en personas que presentan sintomatología depresiva. Por su parte, Lloyd, Malek-Ahmadi, Barclay, Fernández, y Chartrand (2012), analizaron el valor de la inteligencia emocional como factor predictor de depresión, en población mayor. Estos autores encontraron que, por cada punto positivo en inteligencia emocional, el riesgo de desarrollar sintomatología depresiva disminuía un $6 \%$.

A partir de los diferentes estudios, se extrae la idea de que procesos cognitivos y emocionales mantienen una estrecha relación con la salud, concebida ésta desde una perspectiva multidimensional e integradora, en el desarrollo del individuo. Así, autores como FernándezAbascal y Martín-Díaz, (2015), encuentran que las dimensiones de la inteligencia emocional son suficientes para explicar diversos componentes de la salud física y mental, así como para predecir determinados comportamientos relacionados con la salud.

Los datos que se presentan a continuación, vienen a completar los resultados obtenidos en un análisis previo (Pérez-Fuentes et al., 2014), en el que se examinan los cambios en las dimensiones de inteligencia emocional tras la intervención con el PECI-PM. En este caso, se encontraron mejoras en las puntuaciones de las dimensiones Intrapersonal, Interpersonal, Adaptabilidad y Estado de ánimo. Al no encontrarse cambios significativos en la dimensión de Manejo del estrés, se discute el peso de esta dimensión en la inteligencia emocional, en población mayor.

Por la estrecha relación que, tal y como se ha visto, mantienen los aspectos relacionados con la inteligencia emocional y la salud, era de esperar que una mejora en los primeros, revelara cambios en dimensiones propias de la segunda. Por tanto, el objetivo que se plantea con el presente trabajo es analizar los efectos de la primera implementación de un programa de estimulación cognitiva e inteligencia emocional, en la percepción de la salud (física, mental, social, dolor,...), en personas mayores de 65 años.

\section{Método}

\section{Participantes}

La población de estudio estuvo integrada por un total de 67 sujetos que participan en un programa de estimulación cognitiva e inteligencia emocional. Los criterios para la selección de la muestra ( $N$ =28), fueron: completar las 20 sesiones iniciales del programa, y cumplimentar el cuestionario de evaluación, pre-post intervención.

Las características sociodemográficas de los participantes se resumen en la tabla 1 . Se trata de 28 alumnos de la Universidad para Mayores de Almería, con una media de edad de 69.18 años $(D T=6.31)$, siendo el rango de edad de 60-85 años. Del total de la muestra, el $92.9 \%$ son mujeres $(N=26)$, y el $7.1 \%(N$ $=2$ ) hombres, con una media de edad de 69.08 (DT $=6.51)$ y $70.50(D T=3.53)$ años respectivamente. En cuanto al estado civil, el $42.9 \%(N=12)$ son casados, el 39.3\% ( $N=11)$ viudos, el 10.7\% $(\mathrm{N}=3)$ separados/ divorciados, y el $7.1 \%(N=2)$ solteros. Finalmente, según el nivel de estudios, el 14.3\% tiene estudios primarios $(N=4)$, el $17.9 \%$ con Graduado Escolar $(N=5)$, un $32.1 \%$ estudios secundarios $(N=9)$, y el $35.7 \%$ tiene estudios universitarios $(N=10)$.

\section{Instrumentos}

Para la intervención, se aplicó el Programa de Estimulación Cognitiva e Inteligencia Emocional para personas mayores (PECI-PM) (Pérez-Fuentes, Molero, Osorio, \& Mercader, 2014). Este programa, dirigido a personas mayores de 55 años, combina técnicas de estimulación cognitiva y recursos para el entrenamiento de habilidades propias de la inteligencia emocional. Sus objetivos son: mantener y optimizar el rendimiento 
Tabla 1

Distribución de la muestra, según características sociodemográficas

\begin{tabular}{|c|c|c|}
\hline Variables & $N$ & $\%$ \\
\hline \multicolumn{3}{|l|}{ Edad } \\
\hline 60-69 años & 17 & $60.7 \%$ \\
\hline 70 años o más & 11 & $39.3 \%$ \\
\hline \multicolumn{3}{|l|}{ Sexo } \\
\hline Hombre & 2 & $7.1 \%$ \\
\hline Mujer & 26 & $92.9 \%$ \\
\hline \multicolumn{3}{|l|}{ Estado civil } \\
\hline Casado & 12 & $42.9 \%$ \\
\hline Viudo & 11 & $39.3 \%$ \\
\hline Soltero & 2 & $7.1 \%$ \\
\hline Separado/ Divorciado & 3 & $10.7 \%$ \\
\hline \multicolumn{3}{|l|}{ Nivel de estudios } \\
\hline Estudios Primarios & 4 & $14.3 \%$ \\
\hline Graduado Escolar & 5 & $17.9 \%$ \\
\hline Estudios Secundarios & 9 & $32.1 \%$ \\
\hline Estudios Universitarios & 10 & $35.7 \%$ \\
\hline
\end{tabular}

de las personas mayores en tareas que requieren la puesta en marcha de las funciones ejecutivas, así como potenciar el uso de estrategias efectivas en el manejo de las emociones.

El PECI-PM, se desarrolla a través de una aplicación informática desarrollada para Tablet. La tarea del sujeto consiste en responder ante diferentes sesiones de juego, manipulando los cubos que aparecen en la pantalla táctil. El PECI-PM es una herramienta dinámica e interactiva, que proporciona feedback inmediato a las respuestas del jugador.

Para la evaluación de la salud, se administró la adaptación española (Alonso, Prieto, \& Anto, 1995) del Short Form-36 Health Survey (SF-36; Ware \& Sherbourne, 1992). El SF-36, consta de 36 ítems que evalúan estados positivos y negativos de la salud. Incluye 8 escalas (Función física, Rol físico, Dolor corporal, Salud general, Vitalidad, Función social, Rol emocional y Salud mental) e incluye un ítem (Transición de salud) que aporta información sobre la valoración del cambio en la salud actual con respecto al año anterior. Las respuestas se organizan en escalas tipo Likert, con niveles variables (desde dos hasta seis puntos) según el ítem. En todos los casos, la puntuación para cada dimensión se encuentra en un rango de 0 a 100 (donde $0=$ el peor estado de salud, y $100=$ el mejor estado de salud). En este estudio se utiliza la versión estándar, donde se cuestiona al sujeto sobre aspectos relacionados con su salud, haciendo referencia a las cuatro últimas semanas.

En cuanto a la fiabilidad del instrumento, Vilagut et al. (2005) realizan una estimación a partir de diversos estudios que utilizan el SF-36, obteniendo un $\alpha \geq$ .9 para las escalas Función física, Rol físico y Rol emocional y valores $\alpha$ superiores a .7 en el resto de dimensiones. Además, en nuestro país se disponen de valores normativos del SF-36 para población mayor de 60 años (López-García et al., 2003).

\section{Procedimiento}


El estudio se llevó a cabo de acuerdo con la Declaración de Helsinki. Todos los sujetos firmaron un consentimiento informado antes de su participación en el estudio. En la Fase I del PECI-PM (Molero, PérezFuentes, Soler, \& Mercader, 2014), los participantes en el programa deben completar 20 sesiones de 45 minutos dos veces por semana. En esta primera fase, el total de los participantes forman parte del grupo experimental, mientras que, para la segunda fase (sesiones de la 21 a la 40), se incorpora al programa un grupo control. En este trabajo se hace referencia a los datos obtenidos durante la primera fase de implementación del PECIPM, de mayo a julio de 2014.

Para la evaluación, se aplicó una versión digitalizada del cuestionario, integrada en la Tablet. La recogida de datos se llevó a cabo en dos momentos: una primera evaluación antes de iniciar las sesiones de entrenamiento (pre); la segunda evaluación, una vez los sujetos completaron las 20 sesiones (post).

\section{Análisis de datos}

Se utilizó el programa estadístico SPSS.19 para el análisis de los datos. Los parámetros descriptivos fueron realizados mediante análisis de frecuencias. Para los análisis de puntuaciones medias, se aplicó el test de Shapiro-Wilk que lleva a rechazar, en todos los casos, la hipótesis de normalidad $(\mathrm{p}<.05)$, por lo que se utiliza la prueba no paramétrica de Wilcoxon para muestras relacionadas.

\section{Resultados}

\section{Análisis de sobrevivencia}

Para comprobar si existían diferencias que pudieran explicar la permanencia de los participantes en el estudio, se llevó a cabo un análisis de sobrevivencia. Para ello, se tomaron datos disponibles pre-post de las variables sociodemográficas recogidas (edad, sexo, estado civil, nivel de estudios). A partir de los resultados obtenidos, se detectaron diferencias estadísticamente significativas solamente atendiendo a la edad $(t=-2,09$; $p<.05)$, siendo los sujetos que completaron todas las sesiones los de mayor edad $(M=69.03 ; D T=6.24)$ frente a los que no continuaron con el programa de intervención $(M=65.14 ; D T=9.51)$.

\section{Efectos de la intervención}

Tal y como se observa en la tabla 2 , tras las 20 sesiones, las puntuaciones obtenidas para la mayoría de las escalas del SF-36 son más altas, en comparación con las obtenidas al inicio de las sesiones (pre). Estas diferencias entre las puntuaciones pre-post intervención, resultan estadísticamente significativas para Función Física $(Z=-2.45 ; p<.05)$, Dolor Corporal $(Z=-2.02 ; p<.05)$, Vitalidad $(Z=-2.43$;

Tabla 2

Puntuaciones medias para las escalas del SF-36 (pre-post intervención) y puntuación total

\begin{tabular}{|c|c|c|c|c|c|c|}
\hline \multirow{2}{*}{ Escalas SF-36 } & \multicolumn{2}{|c|}{ PRE } & \multicolumn{2}{|c|}{ POST } & \multirow{2}{*}{$Z$} & \multirow{2}{*}{ Sig. } \\
\hline & $M$ & $D T$ & $M$ & $D T$ & & \\
\hline Función Física & 72.50 & 19.97 & 81.80 & 20.91 & $-2.45^{*}$ & .01 \\
\hline Rol Físico & 90.78 & 27.90 & 90.38 & 25.57 & .00 & 1.00 \\
\hline Dolor Corporal & 66.70 & 22.53 & 76.96 & 22.98 & $-2.02^{*}$ & .04 \\
\hline Salud General & 54.50 & 14.85 & 65.22 & 16,29 & -.85 & .39 \\
\hline Vitalidad & 63.04 & 14.90 & 71.73 & 16.24 & $-2.43^{*}$ & .01 \\
\hline Función Social & 83.84 & 18.52 & 90.83 & 15.71 & $-2.10 *$ & .03 \\
\hline Rol Emocional & 78.26 & 37.07 & 80.00 & 37.26 & -.10 & .91 \\
\hline Salud Mental & 67.20 & 15.97 & 74.15 & 14.14 & -1.90 & .05 \\
\hline Total SF-36 & 72.77 & 16.49 & 77.63 & 9.44 & -.53 & .59 \\
\hline
\end{tabular}


$p<.05)$, y Función Social $(Z=-2.10 ; p<.05)$. Para las dimensiones Salud General $(Z=-.85 ; p=.39)$, Rol Emocional $(Z=-.10 ; p=.91)$ y Salud Mental $(Z=-1.90 ; p=.05)$, a pesar de no darse diferencias significativas entre las puntuaciones pre-post, para todos los casos se observa una mejora en la puntuación media obtenida tras las 20 sesiones. Por último, el único factor donde no se aprecia una evolución positiva de la puntuación post intervención, es el Rol Físico, con una media de $90.78(D T=27.90)$ al inicio de las sesiones y de $90.38(D T=25.57)$, al finalizar la intervención con la Fase I del programa.

Por otro lado, los sujetos de la muestra obtienen una puntuación Total SF-36 más elevada tras su participación en el programa (POST: $M=77.63$; $D T=9.44)$, en comparación con los resultados de la evaluación inicial (PRE: $M=72.77 ; D T=16.49$ ). En este caso, las diferencias entre las puntuaciones prepost no resultan estadísticamente significativas $(Z=$ $-.53 ; p=.59)$. Finalmente, en cuanto a la percepción del estado de salud actual con respecto al de hace un año (ítem Transición de Salud), los sujetos de la muestra obtienen una media de $53.12(D T=24.82)$ antes de la intervención, y una media de 54.16 (DT $=15.59)$, tras haber completado las 20 sesiones, no resultando estas diferencias estadísticamente significativas $(Z=-.40 ; p=.68)$.

\section{Discusión y conclusiones}

A partir de los resultados obtenidos, se puede concretar que el entrenamiento de las capacidades cognitivas y de habilidades relacionadas con la inteligencia emocional (Pérez-Fuentes et al., 2014), tienen un efecto positivo en la valoración que hace el sujeto sobre su salud (Zeidner, Matthews, \& Roberts, 2012).

Tras la intervención con el PECI-PM, la valoración de la función física resulta significativamente más positiva que al inicio de las sesiones. En este caso, los participantes presentan una percepción más positiva de su capacidad funcional para la realización de las actividades de la vida diaria, tras haber completado la Fase I del programa. No ocurre lo mismo, cuando se le pregunta por los "problemas con el trabajo u otras actividades cotidianas" (rol físico). Debido a que, lo que se analiza en este caso, son los resultados de la aplicación inicial del programa (20 sesiones), sería necesario esperar a los resultados de la Fase II de implementación, para comprobar si estas diferencias entre Función Física y Rol Físico, se deben a la especificidad de la primera, frente a la valoración más general de la segunda.

Por otro lado, la mejora que se produce en Dolor Corporal apoya la propuesta de otros trabajos, que apuestan por el efecto modulador de las emociones en la percepción/regulación del dolor (Bushnell et al., 2013). Con el PECI-PM, se trabajan un amplio repertorio de emociones, pero se hace especial hincapié en las emociones positivas (Harm et al., 2014). De ahí, que los resultados de la mejora en el procesamiento de emociones positivas se reflejen en una disminución en la sensación de dolor (Rhudy \& Meagher, 2001). Además, este efecto positivo de las emociones sobre la percepción del dolor, en línea con las propuestas de otros autores (Apkarian, 2008; Villemure, \& Bushnell, 2009), puede verse potenciado por su relación con el entrenamiento conjunto de las funciones cognitivas.

Estrechamente relacionado con la percepción del dolor y de las limitaciones que supone, se encuentra, la valoración del nivel de activación y energía que dispone al sujeto para realizar las actividades cotidianas (Vitalidad). Existen estudios que han analizado el impacto positivo de las emociones en la práctica de actividades saludables, como el ejercicio físico (Saklofske et al., 2007), para las que se requiere un cierto grado de activación. Y no solo a nivel físico, sino también la percepción subjetiva de contar con energía suficiente para su práctica (McKenna, 2007).

Por otro lado, de acuerdo con otros autores (Arias \& Iglesias-Parro, 2015; Rimé et al., 1991), el funcionamiento social resulta un factor clave en la mejora de la salud tras la primera intervención con el PECI-PM. A pesar de tratarse de un colectivo de mayores que asiste de forma periódica a clases en la Universidad de Mayores, en muchos casos, han declarado que su participación en el programa ha 
supuesto una mejora en el trato con otros compañeros con los que anteriormente no mantenían relación.

Se pone de manifiesto que la mejora en las capacidades que permitan el uso adecuado de la inteligencia emocional en el funcionamiento social, puede ser una estrategia eficaz para el desarrollo de programas de promoción de la salud (Krawczyk et al., 2009), la reducción de los efectos negativos del estrés (Lakey \& Orehek, 2011), o el desarrollo de la capacidad de recuperación (Armstrong et al., 2011; Ruiz et al., 2015; Schneider et al., 2013).

En este punto, resulta adecuado mencionar la particularidad de la muestra de estudio que, pudiendo esperarse un cumplimiento de los estándares propios de un envejecimiento óptimo (nivel de funcionamiento cognitivo alto, baja probabilidad de enfermar, nivel socioeconómico y educativo superiores al promedio de la población, etc.), realmente los criterios de acceso a los Programas Universitarios para Mayores no interfieren con la heterogeneidad del alumnado. Es decir, no existen limitaciones o requisitos de admisión a estos programas que den lugar a grupos homogéneos, resultando adecuada para una primera implementación del programa, que permita llevar a cabo las adaptaciones oportunas, para su aplicación posterior en otros colectivos.

Finalmente, a pesar de llevarse a cabo solamente la implementación de 20 sesiones con el programa, los resultados obtenidos en esta fase inicial, ya revelan una mejora en la percepción de la salud de los participantes, motivada por el trabajo conjunto sobre cogniciónemoción y reflejada en una puntuación total del SF-36 más positiva. No obstante, aunque en el diseño original del programa ya se anticipaban cambios positivos en algunas de las dimensiones de la salud (Bar-On, 2006; Fernández-Abascal \& Martín-Díaz, 2015), será necesario ampliar la muestra y esperar a la implementación de la Fase II del programa, para comprobar si los resultados en algunas de las dimensiones analizadas resultan significativos. Igualmente, una vez superada la limitación del tamaño muestral, será posible el reporte de resultados sobre las diferencias entre participantes, atendiendo a variables sociodemográficas como el género o la edad, aspectos que han sido de reconocido interés en la literatura científica sobre el tema.

\section{Referencias}

Alonso, J., Prieto, L., \& Anto, J. M. (1995). La versión española del SF-36 Health Survey (Cuestionario de Salud SF-36): un instrumento para la medida de los resultados clínicos. Medicina Clínica, 104, 771-776.

Alonso-Recio, L., Serrano-Rodríguez, J. M., CarvajalMolina, F., Loeches-Alonso, A., \& Martín-Plasencia, P. (2012). Reconocimiento de expresiones faciales de emociones en la enfermedad de Parkinson: una revisión teórica. Revista de Neurología, 54(8), 479-489.

Apkarian, A. V. (2008). Pain perception in relation to emotional learning. Current Opinion in Neurobiology, 18(4), 464-468. doi: 10.1016/j.conb.2008.09.012

Arias, A., \& Iglesias-Parro, S. (2015). La generatividad como una forma de envejecimiento exitoso. Estudio del efecto mediacional de los vínculos sociales. European Journal of Investigation in Health, Psychology and Education, 5(1), 109-120. doi:http://dx.doi. org/10.1989/ejihpe.v1i1.95

Armstrong, A. R., Galligan, R. F., \& Critchley, C. R. (2011). Emotional intelligence and psychological resilience to negative life events. Personality and Individual Differences, 51(3), 331-336. doi: 10.1016/j. paid.2011.03.025

Azpiazu, L., Esnaola, I., \& Sarasa, M. (2015). Capacidad predictiva del apoyo social en la inteligencia emocional en adolescentes. European Journal of Education and Psychology, 8(1), 23-29.

Bar-On, R. (1997). The Emotional Quotient Inventory (EQi): Technical Manual. Toronto: Multi-Health Systems.

Bar-On, R. (2006). The Bar-On Model of EmotionalSocial Intelligence (ESI). Psicothema, 18(suplem. 1), 13-25.

Berking, M., \& Wupperman, P. (2012). Emotion regulation and mental health: recent findings, current challenges, and future directions. Current Opinion in Psychiatry, 25(2), 128-134. doi: 10.1097/ YCO.0b013e3283503669 
Broche, Y., Rodríguez, M., \& Martínez, E. (2014). Memoria de rostros y reconocimiento emocional: generalidades teóricas, bases neurales y patologías asociadas. Actualidades en Psicología, 28 (116), 27-40. doi: http://dx.doi.org/10.15517/ap.v28i116.14890

Bucks, R. S. \& Radford, S. A. (2004). Emotion processing in Alzheimer's disease. Aging and Mental Health, 8(3), 222-232. doi: 10.1080/13607860410001669750

Bushnell, M. C., Ceko, M., \& Low, L. A. (2013). Cognitive and emotional control of pain and its disruption in chronic pain. Nature Reviews Neuroscience, 14(7), 502-511. doi: 10.1038/nrn3516

Cardila, F., Martos, A., Barragán, A. B., Pérez-Fuentes, M. C., Molero, M. M., \& Gázquez, J. J. (2015). Prevalencia de la depresión en España: Análisis de los últimos 15 años. European Journal of Investigation in Health, Psychology and Education, 5(2), 267-279. doi:http://dx.doi.org/10.1989/ejihpe.v5i2.118

Clemente, Y., García-Sevilla, J., \& Méndez, I. (2015). Memoria, funciones ejecutivas y deterioro cognitivo en población anciana. European Journal of Investigation in Health, Psychology and Education, 5(2), 153-163. doi:http://dx.doi.org/10.1989/ejihpe.v5i2.108

Del Barrio, C., Barrios, A., Granizo, L., van der Meulen, K., Andrés, S., \& Gutiérrez, H. (2011). Contribuyendo al bienestar emocional de los compañeros: evaluación del Programa Compañeros Ayudantes en un instituto madrileño. European Journal of Education and Psychology, 4(1), 5-17. doi: 10.1989/ejep.v4i1.73

Delgado, A. (2016). La medida de la comprensión emocional con el modelo de Rasch. Actualidades en Psicología, 30(120), 47-56. doi: http://dx.doi. org/10.15517/ap.v29i119.21516

Díaz-Prieto, C., \& García-Sánchez, J. (2015). Identification of relevant elements for promoting effective interventions in old age / / Identificación de elementos relevantes para promover intervenciones eficaces en la vejez. Revista de Psicodidáctica/ Journal of Psychodidactics, 21(1), 157-173. doi: http://dx.doi. org/10.1387/RevPsicodidact.13854
Fernández-Abascal, E. G., \& Martín-Díaz, M. D. (2015). Dimensions of emotional intelligence related to physical and mental health and to health behaviors. Frontiers in Psychology, 6, 317. http://doi. org/10.3389/ fpsyg.2015.00317

Fernández-Ballesteros, R., Zamarrón, M. D., López, M. D., Molina, M. A., Díez, J., Montero, P., \& Schettini, R. (2010). Envejecimiento con éxito: criterios y predictores. Psicothema, 22(4), 641-647

Garrido-Vásquez, P., Jessen, S., \& Kotz, S. A. (2011). Perception of emotion in psychiatric disorders: On the posible role of task, dynamics, and multimodality. Social Neuroscience, 6(5-6), 515-536. doi: 10.1080/17470919.2011.620771

Gázquez, J. J., Pérez-Fuentes, M. C., \& Carrión, J. J. (2010). Análisis de la Memoria Cotidiana en alumnos del Programa Universitario para Mayores en Almería. European Journal of Education and Psychology, 3(1), 155-165. doi: 10.1989/ejep.v3i1.54

Gázquez, J. J., Pérez-Fuentes, M. C., Fernández, M., González, L., Ruiz, I., \& Díaz, A. (2009). Old-age stereotypes related to the gerontology education: an intergenerational study. European Journal of Education and Psychology, 2(3), 263-273. doi: 10.1989/ejep.v2i3.36

Gázquez, J. J., Pérez-Fuentes, M. C., Lucas, F., \& Yuste, N. (2008). Prevalencia de los trastornos mentales en la población mayor. Anales de Psicología, 24(2), 327333. doi: $10.6018 / 42881$

Gázquez, J. J., Pérez-Fuentes, M. C., Mercader, I., \& Molero, M. M. (2011). Prevalencia de la dependencia funcional en personas mayores. Anales de Psicología, 27(3), 871-876. doi: 10.6018/135641

Goleman, D. (1995). Emotional intelligence. Nueva York: Bantam Books.

Goleman, D. (1998). Working with emotional intelligence. Londres: Bloomsbury Publishing Plc.

Gonzalo, E., \& Pasarín, M. I. (2004). La salud de las personas mayores. Gaceta Sanitaria, 18(1), 69-80.

Gutiérrez-Cobo, M. J., Cabello, R., \& Fernández-Berrocal, P. (2016). The Relationship between Emotional 
Intelligence and Cool and Hot Cognitive Processes: A Systematic Review. Frontiers in Behavioral Neuroscience, 10, 101. http://doi.org/10.3389/fnbeh.2016.00101

Hansenne, M., \& Bianchi, J. (2009). Emotional intelligence and personality in major depression: trait versus state effects. Psychiatry Research, 166(1), 63-68. doi: 10.1016/j.psychres.2008.03.015

Harm, J., Vieillard, S., \& Didierjean, A. (2014). Using humour as an extrinsic source of emotion regulation in young and older adults. The Quarterly Journal of Experimental Psychology, 61(10), 1895-1909. doi: 10.1080/17470218.2013.873474

Hughes, E. K., \& Gullone, E. (2011). Emotion regulation moderates relationships between body image concerns and psychological symptomatology. Body Image, 8(3), 224-231. doi: 10.1016/j. bodyim.2011.04.001

Iglesias-Parro, S., \& Arias Orduña, A. (2015). Aspectos estructurales y funcionales del apoyo social y su impacto en la salud objetiva y percibida de los mayores de gran edad. European Journal of Investigation in Health, Psychology and Education, 5(2), 243-252. doi:http://dx.doi.org/10.1989/ejihpe.v5i2.116

Jacobs, M., Snow, J., Geraci, M., Vythilingam, M., Blair, R. J., Charney, D. S., Pine, D. S., \& Blair, K. S. (2008). Association between level emotional intelligence and severity of anxiety in generalized social phobia. Journal of Anxiety Disorders, 22(8), 1487-1495. doi: 10.1016/j.janxdis.2008.03.003

Jazaieri, H., Morrison, A. S., Goldin, P. R., \& Gross, J. J. (2015). The role of emotion and emotion regulation in social anxiety disorder. Current Psychiatry Reports, 17(1), 531. doi: 10.1007/s11920-014-0531-3

Killgore, W. D., Kahn-Greene, E. T., Lipizzi, E. L., Newman, R. A., Kamimori, G. H., \& Balkin, T. J. (2008). Sleep deprivation perceived emotional intelligence and constructive thinking skills. Sleep Medicine, 9(5), 517-526.

Krawczyk, E., Llek, A., Mroz, S., Kamenczak, A., \& Maj, J. C. (2009). Emotional identification and management disorders among benzodiazepine dependent patients as a factor leading towards interpersonal relations problems. Preeglad Lekarski, 66(6), 319-322.

Lakey, B., \& Orehek, E. (2011). Relational regulation theory: A new approach to explain the link between perceived social support and mental health. Psychological Review, 118(3), 482-495. doi: 10.1037/a0023477

Lazarus, R. S. (1991). Emotion and adaptation. Oxford, UK: Oxford University Press.

LeDoux, J. E. (1996). The emotional brain: The mysterious underpinnings of emotional life. New York: Simon \& Schuster.

Lloyd, S. J., Malek-Ahmadi, M., Fernández, M. R., \& Chartrand, M. S. (2012). Emotional intelligence (EI) as a predictor of depression status in older adults. Archives of Gerontology and Geriatrics, 55(3), 570-573. doi: 10.1016/j.archger.2012.06.004

Lombas, A. S., Martín-Albo, J., Valdivia-Salas, S., \& Jiménez, T. (2014). The relationship between perceived emotional intelligence and depressive symptomatology: The mediating role of perceived stress. Journal of Adolescence, 37(7), 1069-1076. doi: 10.1016/j.adolescence.2014.07.016

Lopesino, P. C., López-Ceron, A. B., Muñoz, M. P., \& Echanagorría, A. M. (2011). Envejecimiento Activo, Libro Blanco. Madrid: Ministerio de Sanidad, Política Social e Igualdad.

López-García, E., Banegas, J. R., Graciani, A., Gutiérrez-Fisac, J. L., Alonso, J., \& RodríguezArtalejo, F. (2003). Valores de referencia de la versión española del Cuestionario de Salud SF-36 en población adulta de más de 60 años. Medicina Clínica, 120(15), 568-573.

Markey, M., \& Vander Wal, J. S. (2007). The role of emotional intelligence and negative affect in bulimic symptomatology. Comprebesive Psychiatry, 48(5), 458-464. doi: 10.1016/j. comppsych.2007.05.006

McKenna, J. (2007). Emotional intelligence training in adjustment to physical disability and illness. International Journal of Therapy and Rehabilitation, 14(12), 551-556. 
Molero, M. M., Pérez-Fuentes, M. C., Soler, F., \& Mercader, I. (2014). Programa de estimulación cognitiva e inteligencia emocional: versión beta del programa de intervención para mayores. En J.J. Gázquez, M.C. Pérez-Fuentes, M.M. Molero, I. Mercader y F.J. Soler (Comps.), Calidad de vida, cuidadores e intervención para la mejora de la salud en el envejecimiento. Volumen II (pp. 289-295). Almería: ASUNIVEP.

Montero, P., Rueda, B., \& Bermúdez, J. (2014). Relación de la personalidad tipo $\mathrm{D}$ y el agotamiento vital con las emociones negativas y el ajuste psicológico a la enfermedad cardíaca. Revista de Psicopatología y Psicología Clínica, 17(2), 93-106.

Organización Mundial de la Salud (2015). Informe Mundial sobre el envejecimiento y la salud. Ginebra: Organización Mundial de la Salud.

Pérez-Fuentes, M. C., Gázquez, J. J., Mercader, I., \& Molero, M. M. (2014). Brief Emotional Intelligence Inventory for Senior Citizens (EQi-M20). Psicothema, 26(4), 524-530. doi: 10.7334/ psicothema2014.166

Pérez-Fuentes, M., Molero, M., Gázquez, J., \& Soler, F. (2014). Estimulación de la Inteligencia Emocional en mayores: El programa PECI-PM. European Journal of Investigation in Health, Psychology and Education, 4(3), 329-339. doi:http:/ / dx.doi.org/10.1989/ejihpe.v4i3.80

Pérez-Fuentes, M. C., Molero, M. M., Mercader, I., Flores, F. S., Barragán, A., Calzadilla, Y., \& Gázquez, J. J. (2015). Salud percibida y salud real: prevalencia en las personas mayores de 60 años. Enfermería Universitaria, 12(2), 56-62. doi:10.1016/j. reu.2015.03.002

Pérez-Fuentes, M. C., Molero, M. M., Osorio, M. J., \& Mercader, I. (2014). Propuesta de intervención cognitiva en personas mayores: Programa de Estimulación Cognitiva e Inteligencia Emocional para Mayores. International Journal of Developmental and Educational Psychology, 1(1), 477-488.
Plaza, M., Requena, C., Rosario, I., \& López, V. (2015). La relación entre el tiempo no estructurado, el ocio $\mathrm{y}$ las funciones cognitivas en personas mayores. European Journal of Education and Psychology, 8(2), 6067. doi:10.1016/j.ejeps.2015.05.001

Quoidbach, J., Mikolajczak, M., \& Gross, J. J. (2015). Positive interventions: An emotion regulation perspective. Psychological Bulletin, 141(3), 655-693. doi:10.1037/a0038648

Ramírez, E., Ortega, A., \& Martos, R. (2015). Las fortalezas en personas mayores como factor que aumenta el bienestar. European Journal of Investigation in Health, Psychology and Education, 5(2), 187-195. doi:http://dx.doi.org/10.1989/ejihpe.v5i2.111

Rhudy, J. L., \& Meagher, M. W. (2001). The role of emotion in pain modulation. Current Opinion in Psychiatry, 14(9), 241-245.

Rimé, B., Mesquita, B., Boca, S., \& Phillippot, P. (1991). Beyond the emotional event: six studies on the social sharing of emotion. Cognition and Emotion, 5(5-6), 435-465. doi: 10.1080/02699939108411052

Ruiz, T., Suengas, A. G., Simón, T., \& Pastor, N. (2015). Is there a deficit in recognition in old age? Psicothema, 27(1), 26-31. doi:10.7334/ psicothema2014.148

Saklofske, D. H., Austin, E., Galloway, J., \& Davidson, K. (2007). Individual difference correlates of health-related behaviours: Preliminary evidence for links between emotional intelligence and coping. Personality and Individual Differences, 42(3), 491-502. doi: 10.1016/j.paid.2006.08.006

Salguero, J. M., Fernández-Berrocal, P., Ruiz-Aranda, D., Castillo, R., \& Palomera, R. (2011). Inteligencia emocional y ajuste psicosocial en la adolescencia: El papel de la percepción emocional. European Journal of Education and Psychology, 4(2), 143-152. doi: $10.1989 /$ ejep.v4i2.84

Salovey, P., \& Mayer, J. D. (1990). Emotional intelligence. Imagination, Cognition y Personality, 9(3), 185-211. 
Sanz de Acedo, M. L., \& Sanz de Acedo, M. T. (2013). How creative potential is related to metacognition. European Journal of Education and Psychology, 6(2), 6981. doi: 10.1989/ ejep.v6i2.104

Schmidt, J. E., \& Andrykowski, M. A. (2004). The role of social and dispositional variables associated with emotional processing in adjustment to breast cancer: an internet-based study. Health Psychology, 23(3), 259-266.

Schneider, T. R., Lyons, J. B., \& Khazon, S. (2013). Emotional intelligence and resilience. Personality and Individual Differences, 55(8), 909-914. doi: 10.1016/j. paid.2013.07.460

Smith, P. J., \& Blumenthal, J. A. (2011). Psychiatric and Behavioral Aspects of Cardiovascular Disease: Epidemiology, Mechanisms, and Treatment. Revista Española de Cardiología (English Edition), 64(10), 924933. doi: 10.1016/j.recesp.2011.06.003

Smith, C. A., \& Lazarus, R. S. (1993). Appraisal components, core relational themes, and the emotions. Cognition and Emotion, 7(3-4), 233-269.

Suzuki, A., \& Akiyama, H. (2013). Cognitive aging explains age-related differences in face-based recognition of basic emotions except for anger and disgust. Aging, Neuropsychology, and Cognition: A Journal on Normal and Dysfunctional Development, 20(3), 253-
270. doi: 10.1080/13825585.2012.692761

Vilagut, G., Ferrer, M., Rajmil, L., Rebollo, P., Permanyer-Miralda, G., Quintana, J. M., Santed, R., Valderas, J. M., Ribera, A., Domingo-Salvany, \& Alonso, J. (2005). El Cuestionario de Salud SF36 español: una década de experiencia y nuevos desarrollos. Gaceta Sanitaria, 19(2), 135-150.

Villemure, C., \& Bushnell, M. C. (2009). Mood influences supraspinal pain processing separately from attention. Journal of Neuroscience, 29(3), 705715. doi: 10.1523/JNEUROSCI.3822-08.2009

Ware, J. E., \& Sherbourne, C. D. (1992). The MOS 36item short-form health survey (SF-36). Conceptual framework and item selection. Medical Care, 30(6), 473-483.

Watson, H. J., Steele, A. L., Bergin, J. L., Fursland, A., \& Wade, T. D. (2011). Bulimic symptomatology: The role of adaptive perfectionism, shape and weight concern, and self-esteem. Behaviour Research and Therapy, 49(9), 565-572. doi: 10.1016/j. brat.2011.04.002

Zeidner, M., Matthews, G., \& Roberts, R. D. (2012). The emotional intelligence, health and well-being nexus: what have we learned and what have we missed? Applied Psychology: Health and Well-Being, 4(1), 1-30. doi:10.1111/j.1758-0854.2011.01062.x

Recibido: 29 de abril de 2016 Aceptado: 7 de setiembre de 2016 
